\title{
Determinación de la longitud de onda máxima por barrido espectral dentro del rango ultravioleta visible (UV-VIS)
}

\section{Length Determination of Maximum Waves by Spectrum Sweep within an Ultraviolet-visible Spectral Region (UV-VIS)}

\author{
Gema Eunice Acosta Niño*, Alejandro Bourdon, Dennis Isabel Guitierrez \\ Universidad Militar Nueva Granada. Grupo de investigación: InQuiBio
}

FEChA DE ENTREGA: 18 DE ENERO DE 2016

FeCha DE EVAluación: 18 DE MARzo DE 2016

FECHA DE APROBACIÓN: 22 DE ABRIL DE 2016

\begin{abstract}
Resumen El presente trabajo hace parte de un proyecto desarrollado en La Universidad Militar Nueva Granada, Bogotá, Colombia, con el se ha elaborado un prototipo para La recuperación de metales provenientes de la pequeña y mediana industria del sector galvánico, específicamente en la ciudad de Bogotá. Como resultado del mismo se ha incursionado en métodos analíticos los cuales permiten determinar la concentración de los metales a retirar en aguas, y su contenido después de haber realizado la electrocoagulación, estos métodos en general requieren del uso de equipos sofisticados para estos análisis, los cuales las pequeñas y medianas industrias no están en capacidad de adquirir. Es bien sabido que en el último siglo se ha generado una gran cantidad de material contaminante en los acuíferos, provenientes del sector agropecuario, aguas domésticas e industria, causando gran afectación a suelos.

Para mitigar el impacto ambiental, se busca cuantificar estos residuos en muestras acuosas controlando concentraciones de vertimientos generados por las industrias. Comúnmente se usa la técnica de absorción atómica en la cuantificación, pero los altos costos de los equipos hacen inviable la absorción atómica para los laboratorios no especializados. Por esta razón, se busca adaptar una técnica analítica en espectrofotométrica visible que permitan determinar $\mathrm{Zn}^{2+}$, para ello es necesario determinar la longitud de onda optima a la cual se debe trabajar en el espectrofotómetro, con las cantidades adecuadas de reactivos que desarrollen un cromóforo apropiado para realizar cada lectura. Dentro de este estudio se analizan parámetros con respecto al tiempo, temperatura y $\mathrm{pH}$, para establecer las mejores condiciones en las cuales se produce el cromóforo, para obtener una lectura adecuada en la absorbancia, cuyo valor es usado para la elaboración de
\end{abstract}

* gema.acosta@unimilitar.edu.co 
la curva de calibración que permita interpolar los valores de contenido de $\mathrm{Zn}^{2+}$, para poder cuantificar.

\begin{abstract}
This paper is part of a project carried out by Universidad Militar Nueva Granada, Bogotá, Colombia, in order to create a prototype for the recovery of metals coming from small and medium-sized enterprises belonging to the galvanic sector in Bogotá. As a result, we used analytical methods that allowed us to determine the metal concentration in the water as well as its content after carrying out the electrocoagulation. Generally, these methods require using sophisticated equipment, and small and medium-sized enterprises cannot afford it. During the last century, a huge amount of polluting material coming from farming, domestic, and industry water has been generated in aquifers, which affects the soil. In order to mitigate the environmental impact, we look to quantify these remains in watery samples by controlling concentrations of wastewater discharges generated by industries. Usually, the atomic absorption technique is used when quantifying, but the high cost of the equipment does not allow non-specialized laboratories to use it. For this reason, we want to adapt an analytic technique in visible spectrophotometric that enables the determination of $\mathrm{Zn}^{2+}$. In order to do this, it is necessary to determine the length of the optimum wage the spectrophometer should use, along with the right amount of reactants to develop the right chromophore for each reading. This study also analyzes parameters regarding time, temperature, and $\mathrm{pH}$ in order to establish the best conditions a chromophore is produced and to obtain an adequate absorbency reading, whose value is used to elaborate the calibration curve that allows interpolating $\mathrm{Zn}^{2+}$ content values in order to quantify.
\end{abstract}

Palabras Clave: cromóforo; absorbancia, calibración.

Keywords: cromophore, absorbency, calibration.

\title{
1. Introducción
}

Como seres humanos un recurso vital para nuestro desarrollo social es el agua potable, del cual hay disponible en la actualidad un 0,025\% del total en todo el planeta, Colombia es el sexto país con mayor potencial hídrico, pero sucede que la mitad de este potencial tiene altos niveles de contaminación, debido a que en el último siglo se ha generado una gran cantidad de material contaminante en los acuíferos. Las principales fuentes de contaminación en el agua potable, son las aguas residuales emanadas por el sector agropecuario, las aguas domésticas y la industria. La última se estima genera nueve mil toneladas de material contaminante. Los mayores contaminantes del agua potable son los hidrocarburos y compuestos con metales pesados como mercurio $(\mathrm{Hg})$, plomo $(\mathrm{Pb})$, cobre $(\mathrm{Cu})$, cromo $(\mathrm{Cr})$, cinc $(\mathrm{Zn})$, selenio $(\mathrm{Se})$ y cadmio $(\mathrm{Cd})$.

Dentro de las industrias que generan estos contaminantes encontramos al sector minero, siderúrgico, petrolero, químico, alimenticio, curtiembres y metalúrgico. Este último es conocido por los altos vertimientos de metales pesados utilizados en recubrimientos que se realizan a superficies metálicas, principalmente con 
$\mathrm{Cr}^{6+}$ y $\mathrm{Zn}^{2+}$, siendo el $\mathrm{Zn}^{2+}$ ambientalmente toxico en bajas concentraciones (mayor a 5 ppm), estipulado por la resolución 1074 de 1997 expedida por el Departamento Administrativo del Medio Ambiente (DAMA) en Colombia.

Para mitigar el impacto ambiental, se busca cuantificar estos residuos en muestras acuosas para controlar las concentraciones de vertimientos generados por las industrias. Comúnmente se usa la técnica de absorción atómica en la cuantificación, pero los altos costos de los equipos hacen inviable la absorción atómica para los laboratorios no especializados. Por esta razón, se busca adaptar una técnica analítica en espectrofotométrica visible que permitan determinar $\mathrm{Zn}^{2+}$, para ello es necesario determinar la longitud de onda optima a la cual se debe trabajar en el espectrofotómetro, con las cantidades adecuadas de reactivos que desarrollen el cromóforo apropiado para realizar una lectura. Dentro de este estudio se analizan parámetros con respecto al tiempo, temperatura y $\mathrm{pH}$, para establecer las mejores condiciones en las cuales se produce el cromóforo, para obtener una lectura adecuada en la absorbancia, cuyo valor es usado para la elaboración de la curva de calibración (esto realizado en otra investigación).

\section{Materiales y métodos}

Para el desarrollo del complejo coloreado (cromóforo) se desarrollaron dos técnicas (cuadros 1 y 2) las cuales fueron evaluadas para elegir el cromóforo más estable a condiciones como: temperatura, $\mathrm{pH}$, exposición a la luz y tiempo.

\begin{tabular}{lc}
\hline Muestra $\left(50 \mathrm{ppm} \mathrm{Zn}^{2+}\right)$ & $300 \mu \mathrm{l}$ \\
Agua & $1900 \mu \mathrm{l}$ \\
Etanol & $300 \mu \mathrm{l}$ \\
Ditizona & $2500 \mu \mathrm{l}$ \\
Volumen total & $5000 \mu \mathrm{l}$ \\
\hline
\end{tabular}

Cuadro 1. Técnicas para el desarrollo del cromóforo. Ensayo 1. El orden de adición de los reactivos es el mismo orden según el cuadro.

Una vez generado los dos métodos se buscó aquel cuya intensidad era perceptible al realizar el barrido espectral en el espectrofotómetro visible marca SPECTRONIC 20 entre los $325 \mathrm{~nm}$ y $1100 \mathrm{~nm}$, determinando la longitud de onda a la cual se logra mayor absorbancia.

De acuerdo a las técnicas para el desarrollo del cromóforo se obtiene alta sensibilidad en el ensayo 1 (Ditizona), ya que genera una intensidad semejante al ensayo 2 (Difenilcarbazida), aunque se encuentra en menor concentración el $\mathrm{Zn}^{2+}$ en el ensayo 1 . 


\begin{tabular}{lc}
\hline Muestra $\left(50 \mathrm{ppm}^{2} \mathrm{Zn}^{2+}\right)$ & $2500 \mu \mathrm{l}$ \\
Agua & $2250 \mu \mathrm{l}$ \\
$\mathrm{NH}_{4} \mathrm{OH}$ & $50 \mu \mathrm{l}$ \\
Difenilcarbazida & $200 \mu \mathrm{l}$ \\
Volumen total & $5000 \mu \mathrm{l}$ \\
\hline
\end{tabular}

Cuadro 2. Técnicas para el desarrollo del cromóforo. Ensayo 2. El orden de adición de los reactivos es el mismo orden según el cuadro.

\section{Resultados y discusión}

A continuación se presentan las evidencias fotográficas del color generado por el cromóforo generado con ditizona sometido a las diferentes variables: tiempo (60 min), luz solar (durante $60 \mathrm{~min})$, temperatura $\left(50{ }^{\circ} \mathrm{C}\right.$ durante $60 \mathrm{~min}$ ) (figura 1) y variación de $\mathrm{pH}$ (figura 2 ).

De acuerdo a la figura 1 no se observa homogeneidad en el color del cromóforo, lo cual indica que este es sensible tanto a la exposición a la luz, a ser sometido a una alta temperatura manejando como constante un tiempo de 50 minutos para cada muestra.

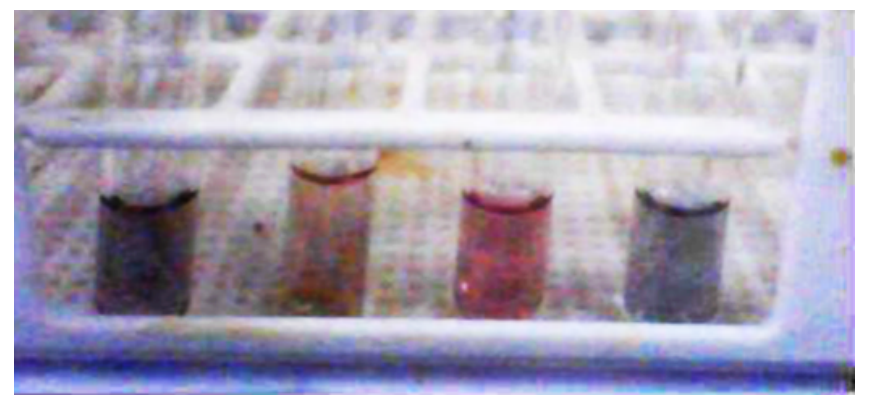

Figura 1. Cambio en el cromóforo de $\mathrm{Zn}^{2+}$ con ditizona sometido a tres variables. a) Cromóforo no expuesto y recién preparado, b) cromóforo expuesto a la luz del sol, c) cromóforo expuesto a una temperatura de $50{ }^{\circ} \mathrm{C}$, d) cromóforo expuesto a la oscuridad y sin temperatura. Cada muestra expuesta a un tiempo de 50 minutos.

Al ser sometida la muestra a pH ácido y básico se confirma la poca estabilidad del cromóforo ya que el color desarrollado vira fuertemente de un $\mathrm{pH}$ a otro, por lo tanto el valor aconsejado puede estar cerca la neutralidad.

Teniendo como referencia esto, el protocolo escogido fue el color desarrollado con la difenilcarbazida ya que este cromóforo no presenta variación de color sometidiendo la muestra a las mismas variables mencionadas anteriormente para el caso de la ditizona. 


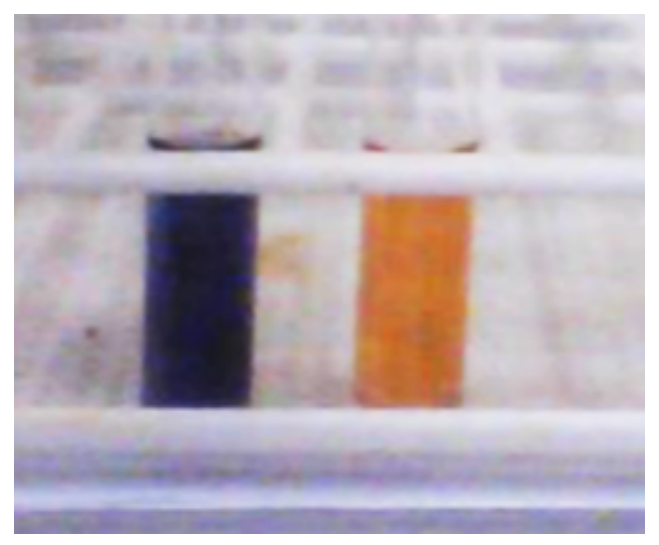

Figura 2. Variación del cromóforo $\mathrm{Zn}^{2+}$ con Ditizona ante el cambio del pH. Lado izquierdo: $\mathrm{pH}$ acido; lado derecho: $\mathrm{pH}$ básico.

Se recomienda tomar lectura inmediatamente se desarrolle el color para mayor precisión en los datos.

Luego de esto, se realiza el barrido espectral (figura 3) en donde se evidencia el máximo de absorbancia en la longitud de onda de $495 \mathrm{~nm}$.

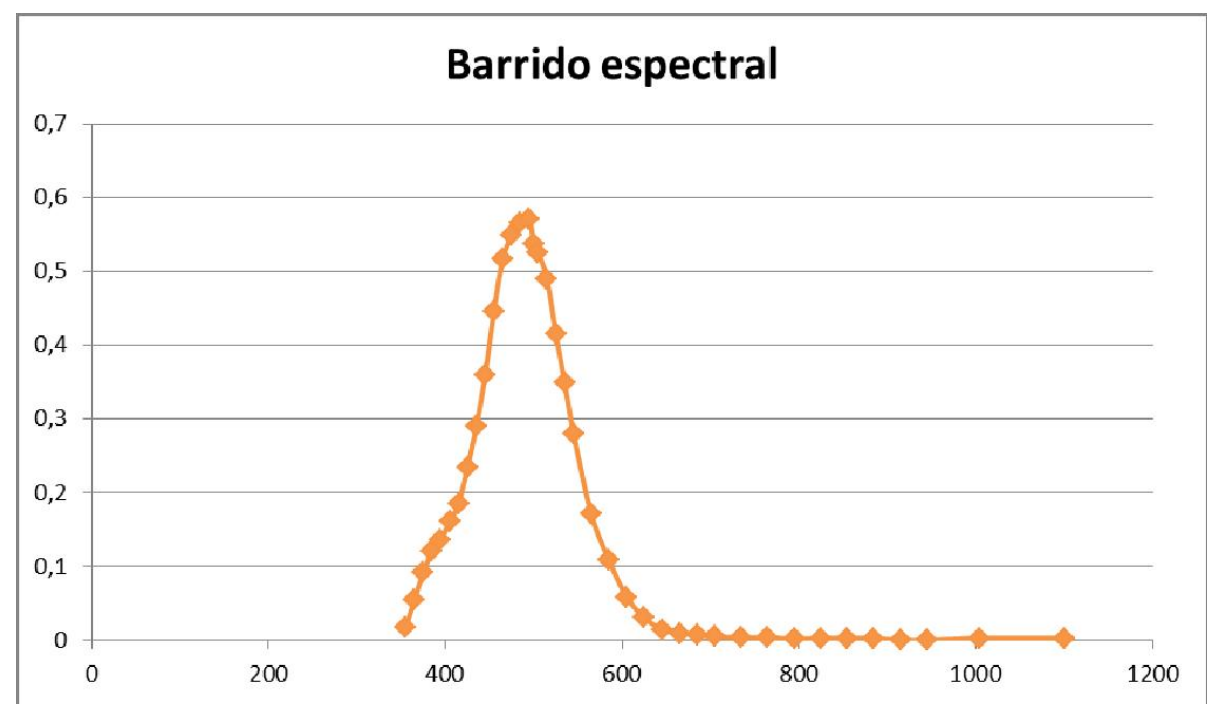

Figura 3. Absorbancia vs. Longitud de onda para cromóforo de $\mathrm{Zn}^{2+}$ y difenilcarbazida. 


\section{Conclusión}

De acuerdo al procedimiento realizado para el desarrollo del cromóforo se determinó que la difenilcarbazida es viable como reactivo para alcanzar una coloración adecuada y hacer lectura a $495 \mathrm{~nm}$.

Este trabajo permite explorar la posibilidad de establecer la presencia de $\mathrm{Zn}^{2+}$ en soluciones acuosas mediante espectrofotometría dentro del rango UV-Vis.

\section{Agradecimientos}

Laboratorios Universidad Militar Nueva Granada

\section{Referencias}

1. DAMA. (1997). Resolución 1074 . Bogotá D.C.

2. Flores Mariños, B. M. (2011). Diseño y validación de un metodo de análisis por espectrofotometría UV-vis para cinc (II) y mercurio (III) en muestras acuosas. Pontificia Universidad Católica del Perú .

3. Kalpakjian, Serope. Schmid, Steven. Manufactura, ingeniería y tecnología. México, 2002. 4ta. Ed. Prentice Hall

4. Yuan, D., Fu, D., Wang, R., \& Yuan, J. (2008). Rapid determination of chromium(VI) in electroplating waste water by use of a spectrophotometric flow injection system. Spectrochimica , 276-279

5. Gonzalez, Ricardo. El 50\% del agua en Colombia es de mala calidad. UN Periódico [en línea]: http://www.unperiodico.unal.edu.co/dper/article/ el-50-del-agua-en-colombia-es-de-mala-calidad.html

6. GREENPEACE. Disponibilidad del recurso. GREENPEACE [en línea]: http: //www .greenpeace.org/colombia/es/campanas/contaminacion/agua/

7. UNAL. Contaminación. UN Periódico [en línea]: http://www.virtual unal.edu. co/cursos/ciencias/2000088/lecciones/seccion4/capitulo10/04_10_04.htm 